\title{
Diffusion-induced dissipation and mode coupling in nanomechanical resonators
}

\author{
Christin Edblom and Andreas Isacsson* \\ Department of Applied Physics, Chalmers University of Technology, S-412 96 Göteborg, Sweden \\ (Received 25 June 2014; revised manuscript received 8 August 2014; published 14 October 2014)
}

\begin{abstract}
We study a system consisting of a particle adsorbed on a carbon nanotube resonator. The particle is allowed to diffuse along the resonator, in order to enable study of, e.g., room-temperature mass sensing devices. The system is initialized in a state where only the fundamental vibration mode is excited, and the ring-down of the system is studied by numerically and analytically solving the stochastic equations of motion. We find two mechanisms of dissipation, induced by the diffusing adsorbate. First, short-time correlations between particle and resonator motions means that the net effect of the former on the latter does not average out, but instead causes nonexponential dissipation of vibrational energy. For vibrational amplitudes that are much larger than the thermal energy this dissipation is linear; for small amplitudes the decay takes the same form as that of a nonlinearly damped oscillator. Second, the particle diffusion mediates a coupling between vibration modes that opens a new dissipation channel by enabling energy transfer from the fundamental mode to the excited modes, which rapidly reach thermal equilibrium.
\end{abstract}

DOI: 10.1103/PhysRevB.90.155425

PACS number(s): 85.85.+j, 05.40.-a, 68.43.Jk

\section{INTRODUCTION}

Nanoelectromechanical (NEM) resonators hold great promise for applications in inertial mass sensing [1,2]. Carbon nanotubes (CNTs) in particular are suited when striving for high sensitivity $[3,4]$, due to their extremely low mass. Recently, using CNT resonators, yoctogram sensitivity was achieved in experiments [5]. In mass sensing applications, it is commonly assumed that an adsorbate, once attached to the surface, remains in the same positions during the time of measurement. However, at elevated temperatures thermal fluctuations can cause the adsorbate to change its position along the tube via diffusion. As the resonant frequency of the system depends on the position of the adsorbate, this gives rise to frequency fluctuations with accompanying phase noise.

For driven resonators, the effect of such frequency fluctuations has recently been studied both theoretically [6-9] as well as experimentally [10]. The effect manifests in a broadening and/or changed shape of the resonant response. However, broadening also arises from dissipation of mechanical energy. Dissipation and the origin of $Q$-factor limitations in nanoelectromechanical systems has been a long-standing research topic where there are still unresolved issues [11-14]. Recently, the connection between dissipation and nonlinear phenomena in NEM resonators has begun to attract attention. This is partly because of the presence of nonlinear damping $[15,16]$ in carbon nanoresonators, and partly due to the recognition that geometric nonlinearites themselves give rise to dissipation [17] and spectral broadening [18]. While it was shown in Refs. [6,8] that a diffusing particle on an otherwise linear resonator induces both spectral broadening as well as a nonlinear response to driving, we also expect the same mechanism to give rise to dissipation and mode coupling. In that case, two questions arise: in what manner does the system relax to equilibrium, and what is the effect of the mode coupling on the the system dynamics? In the present study, we investigate the characteristics of the dissipation process

\footnotetext{
*andreas.isacsson@chalmers.se
}

induced by the diffusing adsorbate in order to answer these questions.

For concreteness, we model a diffusing adsorbate along a carbon nanotube resonator and study the decay of mechanical energy in the system. In order to isolate the dissipative contribution to the resonance broadening, we simulate ring-down measurements such as the ones performed in Refs. [19,20]. Our model is a doubly clamped one-dimensional resonator constrained to move in the plane. A small mass is adsorbed on the resonator, and allowed to diffuse along it as shown in Fig. 1. The resonator is excited in its lowest flexural vibration mode, by means of, e.g., a nearby gate, and the subsequent free evolution of the system is studied.

An example of the distribution of mechanical energy between flexural modes during a simulated ring-down experiment is shown in Fig. 2. A nonexponential decay of the mechanical energy of the fundamental mode is evident; eventually thermal equilibrium is reached. In Sec. III we show that, in the limit of a single flexural mode, the observed decay can be divided into two distinct regimes. In the first regime the vibration amplitude is large, the adsorbate is trapped at an antinode of the vibration, and the mechanical energy decays linearly. In the second regime the amplitude is small and the adsorbate diffuses freely along the nanotube, which exhibits nonexponential dissipation characteristic of nonlinearly damped resonators. This damping is due to the fact that the inertial force acting on the particle causes its motion to have frequency components twice that of the fundamental mode. Because of retardation, this short-time correlation between adsorbate motion and resonator motion causes dissipation, an effect also seen in molecular dynamics studies on graphene resonators [21]. In addition, as the particle changes position, a mode coupling is induced that opens a new channel of dissipation, allowing the transfer of energy to higher-lying modes and causing them as well to equilibrize. As we discuss in Sec. IV, this new dissipation channel means that while the results of Sec. III are qualitatively robust, a quantitative error arises; the rate of decay due to the mode coupling is linearly proportional to the number of higher modes 


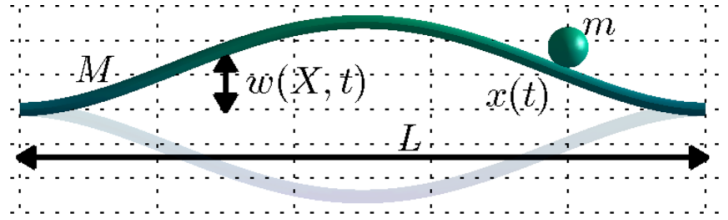

FIG. 1. (Color online) A particle of mass $m$ is adsorbed on a one-dimensional resonator of mass $M$ and length $L$. The transverse displacement of the resonator at coordinate $X$ along the nanotube axis is $w(X, t) ; x(t)$ is the position of the adsorbed particle. The resonator is initialized in its fundamental vibration mode, and the effect of the stochastically diffusing particle on the ring-down of the resonator is studied.

(up to a parameter-dependent cutoff), and hence their total energy. This result is analogous to those found in Refs. [17,18] when introducing conservative geometric nonlinearities in clean nanoresonators.

\section{EQUATIONS OF MOTION FOR A RESONATOR WITH A DIFFUSING PARTICLE}

As shown in Fig. 1, we consider a resonator of length $L$ with mass $M=L \rho$ and bending rigidity $\kappa$. Neglecting longitudinal displacement, the Lagrangian density for the unperturbed resonator is [22]

$$
\mathcal{L}_{0}=\frac{1}{2} \rho \dot{w}^{2}-\frac{1}{2} \sigma w_{X}^{2}-\frac{1}{2} \kappa w_{X X}^{2} .
$$

Here, $w=w(X, t)$ is the transverse displacement (see Fig. 1), $X$ is the coordinate measured along the resonator, and $w_{X}=$ $\partial w / \partial X$. In the limit of small vibration amplitude and/or large prestrain, the built-in tension $\sigma$ can be assumed independent of $w$. The unpertubed eigenfrequencies $\omega_{n}$ and eigenmodes $\phi_{n}(X)$ are found from solving the corresponding equation of motion; see Appendix A. For convenience, we will work with eigenmodes normalized so that $\int d x \phi_{n} \phi_{m}=L \delta_{n m}$, and with boundary conditions corresponding to a doubly clamped beam: $w(0)=w(L)=w_{X}(0)=w_{X}(L)=0$.

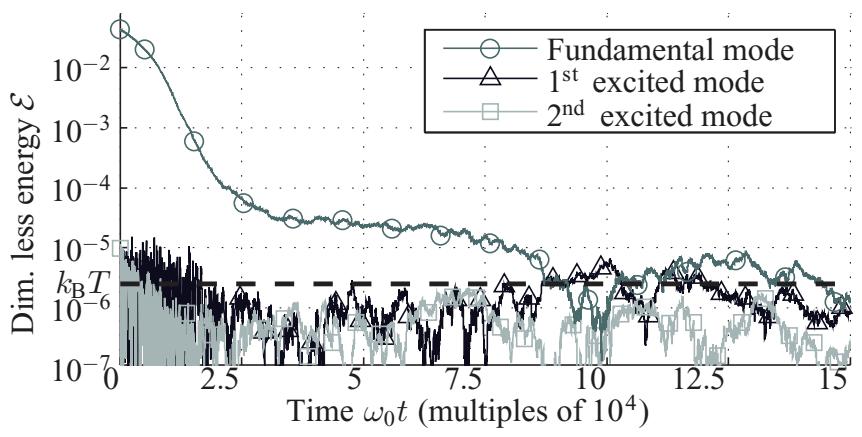

FIG. 2. (Color online) Dimensionless mode energies as function of time during ring-down of the fundamental mode. Here $\epsilon \simeq 10^{-2}$ and $T=500 \mathrm{~K}$. For clarity, only the lowest lying flexural modes are shown; higher modes behave similarily. The dashed line indicates the thermal energy in dimensionless units. The system is initialized in a state where all energy $\left(\mathcal{E}_{0}(0) \simeq 10^{4} k_{\mathrm{B}} T\right)$ is in the fundamental mode, and then allowed to evolve freely. As can be seen, the effect of the particle diffusion is to damp out the fundamental mode and establish equilibrium with higher-lying modes.
Including the adsorbate of mass $m=\epsilon M$ at $X=x(t)$, the total Lagrangian is

$$
\mathcal{L}=\mathcal{L}_{0}+\frac{1}{2} m \delta(x-X)\left[\dot{x}^{2}+\left(\dot{w}+\dot{x} w_{X}\right)^{2}\right] .
$$

Here, the term added to the resonator Lagrangian density $\mathcal{L}_{0}$ is the kinetic energy $\frac{1}{2} m \dot{\mathbf{r}}^{2}$ of the adsorbed particle, where its position $\mathbf{r}(t)=(x(t), w(x(t), t))$.

We expand the displacement in eigenmodes, $w(X, t)=$ $\sum_{n} q_{n}(t) \phi_{n}(X)$, and by variation of $\mathcal{L}$ we find the equations of motions

$$
\begin{aligned}
\ddot{q}_{n}+\omega_{n}^{2} q_{n}-\epsilon \phi_{n}(x) \sum_{k} \omega_{k}^{2} q_{k} \phi_{k}(x) & =0, \\
\dot{x}-\frac{1}{\gamma} \sum_{k, \ell} \omega_{k}^{2} q_{k} q_{\ell} \phi_{k} \phi_{\ell}^{\prime} & =\sqrt{D} \eta(t) .
\end{aligned}
$$

In order to allow for the thermal diffusion of the adsorbate, a stochastic force has been introduced in the right-hand side of Eq. (4). By the fluctuation-dissipation theorem, this force is accompanied by a damping rate $\gamma$. Thus, $\eta(t)$ is a $\delta$-correlated Gaussian noise, i.e., $\left\langle\eta(t) \eta\left(t^{\prime}\right)\right\rangle=\delta\left(t-t^{\prime}\right)$, and $D=2 k_{\mathrm{B}} T / m \gamma$. Throughout, we assume reflecting boundary conditions for the diffusing particle. Finally we note that Eqs. (3) and (4) are derived using the approximation $\partial_{t}^{2} w(x(t), t) \approx-\sum_{n} \omega_{n}^{2} q_{n}(t) \phi_{n}(x)$, which is equivalent to claiming that the effect of the added mass is a small correction to the unperturbed motion, as well as an assumption of strong damping $(m \ddot{x} \ll m \gamma \dot{x})$ that allows the inertial term to be neglected.

The nonlinear system of Eqs. (3) and (4) is numerically integrated using a second-order algorithm [23]. The system is initialized in a state where all energy is stored in the fundamental mode, $q_{n}(t=0)=0, n>0$, and the particle is adsorbed at $x=L / 2$. The resonator dimensions have been chosen to be experimentally realistic: length $1 \mu \mathrm{m}$, diameter $5 \mathrm{~nm}$, and fundamental resonant frequency $\omega_{0}=2 \pi \times 108 \mathrm{MHz}$. Some results are shown in Figs. 2 and 3 , using parameters $\mathcal{D}=$ $D / \omega_{0} L^{2}=2.85 \times 10^{-4}, \epsilon=1.82 \times 10^{-2}$ (corresponding to an adsorbate mass $m$ similar to that of a mid-sized protein molecule or a larger collection of noninteracting smaller adsorbates), and $\gamma=0.241 \omega_{0}$. The initial amplitude $q_{0}$ is here chosen such that $\mathcal{E}_{0}(t=0)=0.044$, where $\mathcal{E}_{n}=\omega_{n}^{2} q_{n}^{2} / \omega_{0}^{2} L^{2}$ is the dimensionless kinetic energy stored in mode $n$.

The main features to note are the initially linear decay of energy in the fundamental mode, the initial trapping of the adsorbate near the center of the resonator followed by diffusion along the length, and the eventual thermalization of all vibration modes. Also note that the higher-lying modes reach an internal equilibrium very rapidly compared to the slow decay of the fundamental mode energy. These results are further discussed in Sec. IV.

As illustrated in Fig. 3, one can identify two distinct limiting cases. The first, high-amplitude limit is characterized by the particle being trapped at the antinode of the vibration around $x \approx L / 2$. In this regime, the energy of the resonator decays linearly in time. As the amplitude of the resonator vibrations decreases, thermal fluctuations overcome the inertial trapping potential, the particle starts to diffuse along the entire length of the nanotube, and the decay rate is no longer linear. 


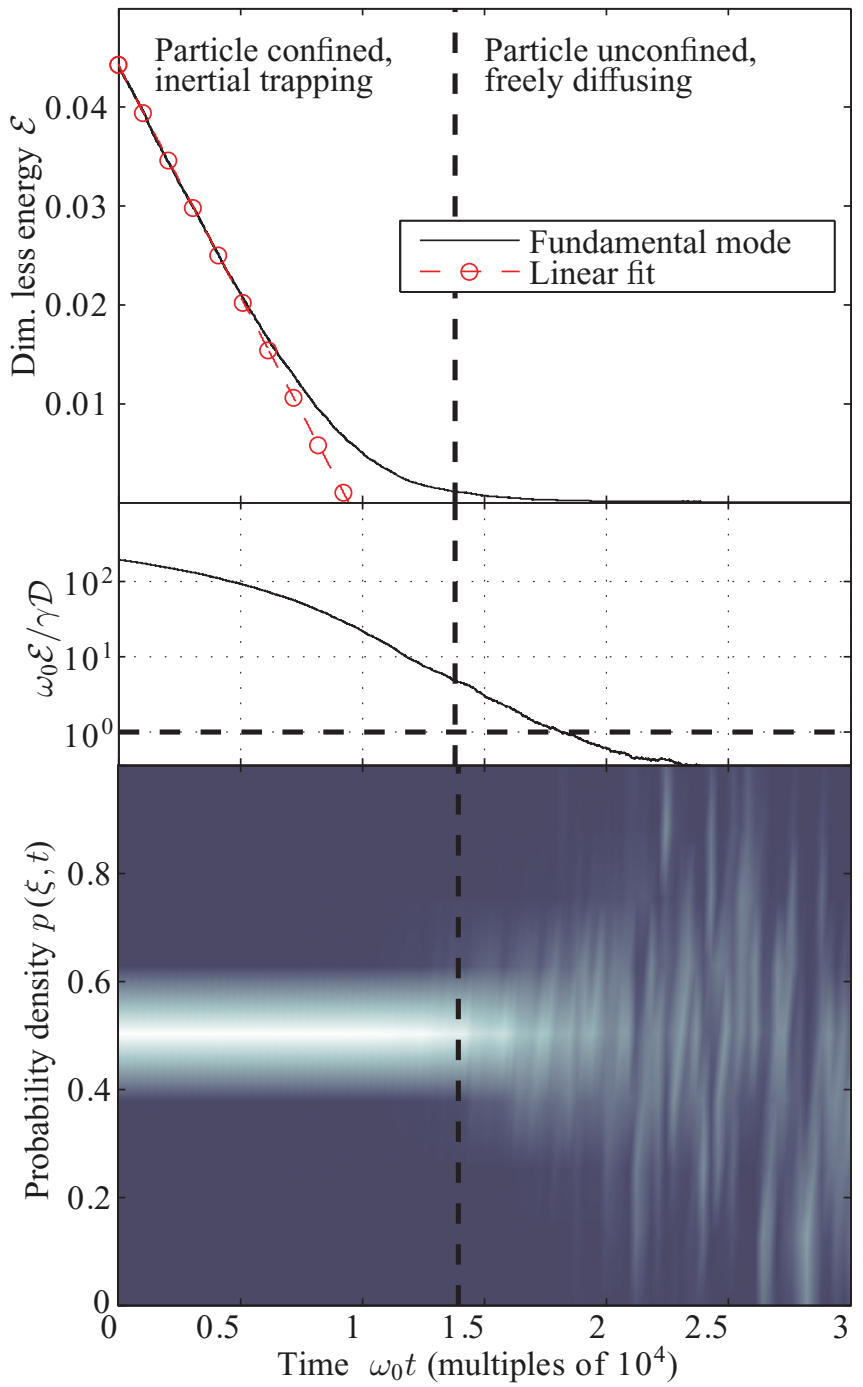

FIG. 3. (Color online) Top: fundamental mode energy as a function of time, together with a linear fit to the initial decay. Center: the parameter $\omega_{0} \mathcal{E} / \gamma \mathcal{D}$, shown below to govern the qualitative behavior of the system, as a function of time. The horizontal dashed line indicates where $\omega_{0} \mathcal{E}=\gamma \mathcal{D}$. Bottom: evolution of the probability distribution $p(\xi, t)$, where $\xi=x / L$, for the particle to be at a certain position $0<\xi<1$ along the resonator as a function of time. For large initial amplitudes, the particle remains inertially trapped at the antinode of the vibration around $\xi \approx 0.5$. The corresponding energy decay is linear in time. As the thermal fluctuations overcome the inertial trapping potential, the particle diffuses freely, and the energy decays algebraically towards equilibrium.

The regime is determined by the parameter $\omega_{0} \mathcal{E}_{0} / \gamma \mathcal{D}=$ $\frac{1}{2} m \omega_{0}^{2} q_{0}^{2} / k_{\mathrm{B}} T$, the ratio between vibrational and thermal energy of the adsorbate. The inertial trapping potential is proportional to the vibrational energy, so the particle remains confined as long as $\omega_{0} \mathcal{E}_{0} / \gamma \mathcal{D} \gtrsim 1$, and diffuses freely when $\omega_{0} \mathcal{E}_{0} / \gamma \mathcal{D} \lesssim 1$. This parameter is shown as a function of time in the center panel of Fig. 3, illustrating the agreement between the value of $\omega_{0} \mathcal{E}_{0} / \gamma \mathcal{D}$ and the behavior of the adsorbate.

The diffusion constant $D$ depends on the adsorbate and resonator materials. While measurements of the diffusion constants of several elements on graphite exist it is not clear that these are applicable to diffusion along a nanotube. The values for diffusion constants and adsorbate masses used in the simulations were chosen to provide good numerical stability, facilitate comparison with results obtained from perturbation theory, and to visualize the different regimes as clearly as possible. Interestingly, as will be seen in Sec. III, the leading relevant parameters that determine the ring-down dynamics in the pertubative regimes are the dimensionless ratios $\epsilon \mathcal{D} \gamma / \omega_{0}$ [trapping regime, Eq. (10)] and $\epsilon \omega_{0} / \gamma$ [free diffusion regime, Eq. (13)]. Since we have $\mathcal{D} \gamma=2 k_{\mathrm{B}} T / m \omega_{0} L^{2}$ by virtue of the fluctuation-dissipation theorem, the precise value of the diffusion constant is not crucial in the trapping regime, provided $D \gg\left(L / q_{0}\right)^{2}\left(k_{\mathrm{B}} T / m \omega_{0}\right)$. The ratio $\epsilon \omega_{0} / \gamma$ for the free diffusion regime becomes $\epsilon m \omega_{0} L^{2} D / 2 k_{\mathrm{B}} T$. Since $D$ is expected to depend exponentially on temperature, probing a large parameter range can thus be done by varying $T$.

\section{SINGLE-MODE DYNAMICS}

To understand the observed energy decay we first focus on a single flexural mode. This simplification of the equations of motion is motivated by simulations, which have not shown any qualitative dependence on the number of included modes. That is, even when only the fundamental mode of the resonator is included, the two regimes identified in Fig. 3 are evident. Below, we will treat the two regimes separately, beginning with the large amplitude case.

Considering only the fundamental mode, i.e., $w(X, t)=$ $q_{0}(t) \phi_{0}(X)$, one finds

$$
\begin{aligned}
& \ddot{q}_{0}+\omega_{0}^{2}\left[1-\epsilon \phi_{0}^{2}(x)\right] q_{0}=0, \\
& \dot{x}=\frac{\omega_{0}^{2}}{2 \gamma} q_{0}^{2} \partial_{x} \phi_{0}^{2}(x)+\sqrt{D} \eta(t) .
\end{aligned}
$$

We measure time in units of $\omega_{0}^{-1}$, and change to action angle variables $(\mathcal{E}(t), \theta(t))$ via the transformations $q_{0}(t)=$ $L \sqrt{\mathcal{E}} \cos \left(\omega_{0} t+\theta\right)$ and $\dot{q}_{0}(t)=-\omega_{0} L \sqrt{\mathcal{E}} \sin \left(\omega_{0} t+\theta\right)$. Then, the equations take the form

$$
\begin{aligned}
& \partial_{\tau} \mathcal{E}=-\epsilon \phi_{0}^{2} \mathcal{E} \sin 2 \nu, \quad(v=\theta+\tau), \\
& \partial_{\tau} \theta=-\epsilon \phi_{0}^{2} \cos ^{2} \nu, \\
& \partial_{\tau} \xi=\frac{\omega_{0}}{2 \gamma} \mathcal{E} \cos ^{2} \nu \partial_{\xi} \phi_{0}^{2}+\sqrt{\mathcal{D}} \eta(\tau),
\end{aligned}
$$

where $\xi=x / L, \tau=\omega_{0} t$ and $\mathcal{D}=D / \omega_{0} L^{2}$. Thus, it is quite clear from Eq. (7) that performing a rotating wave approximation here leads to $\partial_{\tau} \mathcal{E}=0$ and that the effect of the particle diffusion is only to cause fluctuations in resonant frequency. Hence, in order for $\partial_{\tau} \mathcal{E} \neq 0, \xi$ must contain a frequency component $\sin 2 v$ which arises from the first term in Eq. (9). It follows that the observed decay in energy stems from short-time correlations with frequency $2 \omega_{0}$ between particle and resonator motions.

\section{A. Large amplitude vibrations, confined particle}

When $\omega_{0} \mathcal{E} / \gamma \mathcal{D} \gg 1$, the thermal fluctuations cannot overcome the inertial trapping potential and the adsorbate fluctuates around the antinode of the flexural mode. In this regime, the phase noise is typically small and can be neglected when esti- 
mating the decay rate. Furthermore, as the particle is at all times in the vicinity of the antinode we can make the approximation $\partial_{\xi} \phi_{0}^{2} \approx 2 \phi_{0}(0) \phi_{0}^{\prime \prime}(0) \xi=-k \xi$, which renders the diffusion equation (9) linear. Solving for the particle motion yields

$$
\xi(\tau)=\sqrt{\mathcal{D}} \int^{\tau} d \tau^{\prime} \eta\left(\tau^{\prime}\right) e^{-\left(\omega_{0} k / 2 \gamma\right) \int_{\tau^{\prime}}^{\tau} d \tau^{\prime \prime} \mathcal{E}\left(\tau^{\prime \prime}\right) \cos ^{2} v^{\prime \prime}} .
$$

Inserting back into the equation for $\mathcal{E}$, omitting the term vanishing upon averaging over fast fluctuations, and assuming $\mathcal{E}$ to be slow, one finds

$$
\partial_{\tau} \mathcal{E} \approx \frac{1}{2} \epsilon \mathcal{D} k \mathcal{E} \sin 2 \tau \int_{-\infty}^{\tau} d \tau^{\prime} e^{-\left[k \omega_{0} \mathcal{E}(\tau) / 2 \gamma\right] \int_{\tau^{\prime}}^{\tau} d \tau^{\prime \prime} \cos ^{2} \tau^{\prime \prime}} .
$$

Averaging over fast oscillations,

$$
\partial_{\tau} \mathcal{E} \approx \epsilon \mathcal{D} k \mathcal{E} I\left(\frac{k \omega_{0} \mathcal{E}(\tau)}{\gamma}\right)
$$

where the integral $I$ is defined as

$$
I(x)=\frac{1}{2 \pi} \int_{0}^{2 \pi} d \tau \sin 2 \tau \int_{-\infty}^{\tau} d \tau^{\prime} e^{-x \int_{\tau^{\prime}}^{\tau} d \tau^{\prime \prime} \cos ^{2} \tau^{\prime \prime}} .
$$

The integral is well approximated by the expression $I(x) \approx$ $-\{4+\sqrt{\pi}[(x / 2) \operatorname{coth}(x / 2)-1]\}^{-1}$. Hence, for large amplitudes such that $k \omega_{0} \mathcal{E}(\tau) / \gamma \gg 1$, the decay becomes linear, i.e.,

$$
\partial_{\tau} \mathcal{E} \approx-\epsilon \frac{2 \mathcal{D} \gamma}{\sqrt{\pi} \omega_{0}} .
$$

Comparisons with simulation show that this result is correct within an order of magnitude, even when excited modes are included; see Fig. 6. However, as discussed in Sec. IV, adding excited modes introduces more channels of decay, and consequently increases the decay rate.

\section{B. Small amplitude vibrations, unconfined particle}

If $\omega_{0} \mathcal{E} /(\gamma \mathcal{D}) \ll 1$, the system (7)-(9) can be solved by means of perturbation theory. This limit can be seen to be equivalent to the assumption that the vibration amplitude be small enough that the particle is not inertially trapped at an antinode, and falls in the typical parameter regime encountered in most experimental situations. As an example, for a single $\mathrm{Kr}$ atom on a $100-\mathrm{MHz} \mathrm{CNT}$ resonator vibrating with an amplitude of $q_{0}=3 \mathrm{~nm}$ at $T=1 \mathrm{~K}$, one has $\omega_{0} \mathcal{E} / \gamma \mathcal{D}=$ $E_{\mathrm{vib}} / k_{\mathrm{B}} T \approx 0.03$

The corresponding Fokker-Planck equation (FPE) for the distribution function $p(\xi, E, \nu, \tau)$ reads [24]

$$
\begin{aligned}
{\left[\partial_{\nu}+\right.} & \left.\partial_{\tau}\right] p(\xi, E, \nu, \tau) \\
= & \epsilon \phi_{0}^{2} \mathcal{E} \sin (2 \nu) \partial_{\mathcal{E}} p+\frac{\epsilon \phi_{0}^{2}}{2}[1+\cos (2 \nu)] \partial_{\nu} p \\
& -\frac{\omega_{0} \mathcal{E}}{4 \gamma}[1+\cos (2 \nu)] \partial_{\xi}\left[p \partial_{\xi} \phi^{2}\right]+\frac{\mathcal{D}}{2} \partial_{\xi}^{2} p .
\end{aligned}
$$

As noted above, the dissipation of mechanical motion stems from the correlation between the motion of the particle and the resonating beam, induced by the last term in Eq. (11). These correlations occur on a time scale $\omega_{0}^{-1}$ which is much shorter than the scale of the rate of change of energy. Hence, we can find the dissipation rate by making a separation ansatz for fast and slow time scales by the approximation $p \approx p_{0}(\mathcal{E}, \tau) p_{1}(\mathcal{E}, \nu, \xi)$ (a more formal derivation is found in Appendix B). This decouples Eq. (11) into one equation for slow time scales and one for fast time scales, where particle position is described by the latter:

$$
\partial_{\nu} p_{1}=-\frac{\omega_{0} \mathcal{E}}{4 \gamma}[1+\cos (2 v)] \partial_{\xi}\left[p_{1} \partial_{\xi} \phi^{2}\right]+\frac{\mathcal{D}}{2} \partial_{\xi}^{2} p_{1} .
$$

Assuming $E_{\mathrm{vib}} / k_{\mathrm{B}} T \ll 1$ one finds to first order in $\mathcal{E}$ the steady-state solution

$$
p_{1}=1+\frac{\omega_{0} \mathcal{E}}{\mathcal{D} \gamma} \sum_{n} \lambda_{n} f_{n} \frac{\lambda_{n} \cos 2 v+4 \sin 2 v}{\lambda_{n}^{2}+16} \cos (n \pi \xi),
$$

where $\lambda_{n}=\mathcal{D} n^{2} \pi^{2}$ and $f_{n}=\int_{0}^{1} d \xi \cos (n \pi \xi) \phi_{0}^{2}(\xi)$. Inserting this solution into the FPE (11), and integrating over position $\xi$ and the fast variable $v$ yields

$$
\partial_{\tau} p_{0}(\mathcal{E}, \tau)=\epsilon \frac{2 \omega_{0}}{\mathcal{D} \gamma} \sum_{n} \frac{\lambda_{n} f_{n}^{2}}{\lambda_{n}^{2}+16} \partial_{\mathcal{E}}\left(\mathcal{E}^{2} p_{0}\right) .
$$

The solution to this equation is $p(\mathcal{E}, \tau)=\mathcal{E}^{-2} f\left(\frac{1-\alpha \mathcal{E} \tau}{\mathcal{E}}\right)$, where

$$
\alpha=\epsilon \frac{m \omega_{0}^{2} L^{2}}{k_{\mathrm{B}} T} \sum_{n} f_{n}^{2} \frac{\lambda_{n}}{16+\lambda_{n}^{2}} .
$$

If $p(\mathcal{E}, 0)=\delta\left(\mathcal{E}-\mathcal{E}_{0}\right)$ the ensemble averaged energy $\langle\mathcal{E}\rangle$ decays without dispersion and one obtains the characteristic ring-down of a nonlinearly damped oscillator,

$$
\langle\mathcal{E}(\tau)\rangle=\frac{\mathcal{E}_{0}}{1+\alpha \mathcal{E}_{0} \tau} .
$$

This expression does indeed agree well with simulation in the parameter space where perturbation theory is valid; see Fig. 4.

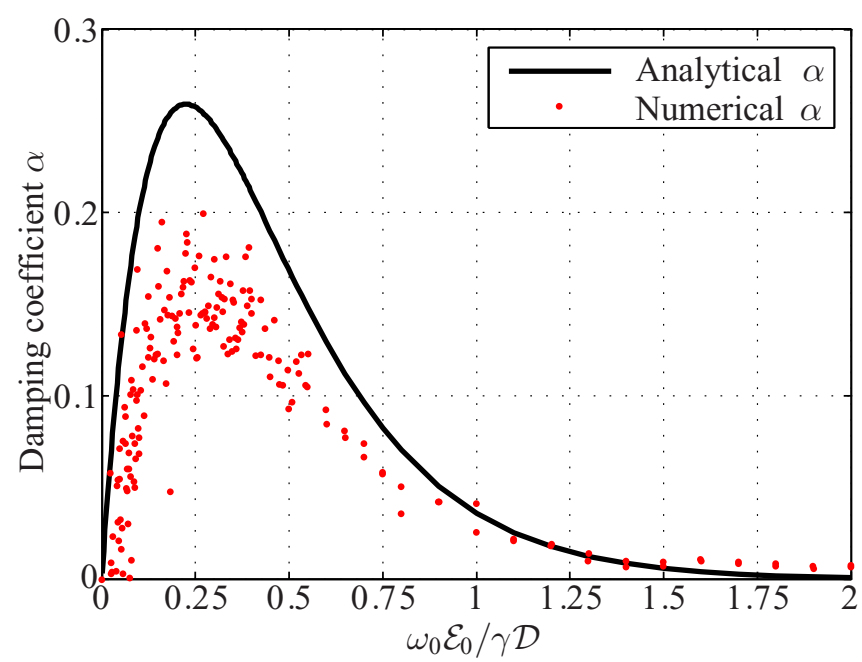

FIG. 4. (Color online) Damping parameter $\alpha$, as calculated by Eq. (14) (solid line) and by a numerical fit (dots). The same simulation parameters as in Fig. 2 were used, with the exception of the initial amplitude, here $\mathcal{E}_{0}(0)=10^{-4}$. The ratio $\omega_{0} \mathcal{E}_{0} / \gamma \mathcal{D}$ was varied by changing the simulation temperature. We see that the perturbative approach is indeed valid for $\omega_{0} \mathcal{E}_{0} / \gamma \mathcal{D} \ll 1$. In the intermediate region $\omega_{0} \mathcal{E}_{0} / \gamma \mathcal{D} \lesssim 1$, Eq. (14) overestimates the magnitude of the damping, but captures the overall shape of the curve and the location of the maximal damping. 


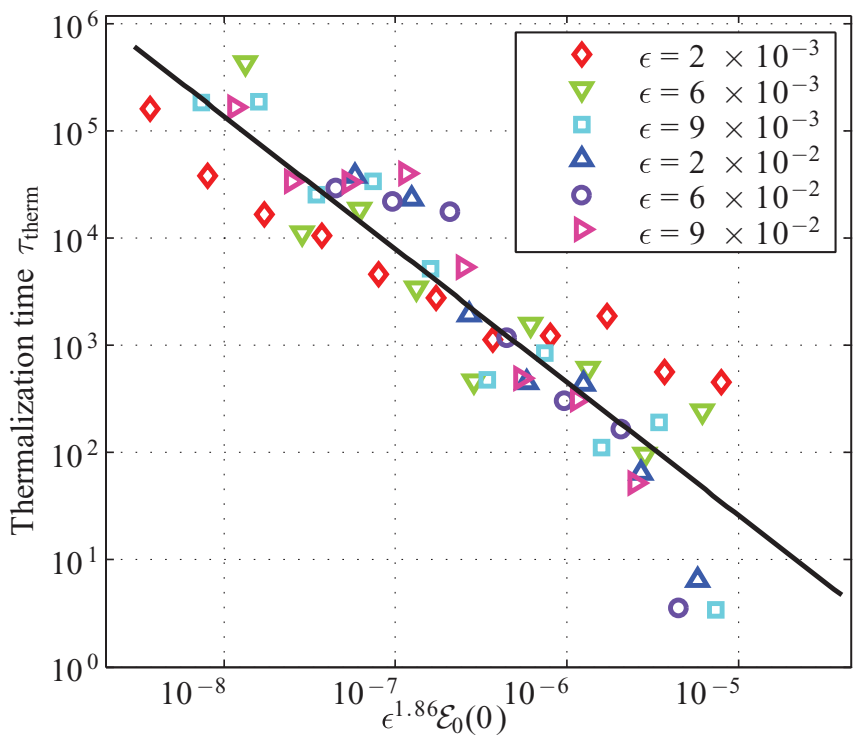

FIG. 5. (Color online) Dependence of the thermalization time (the time at which $\mathcal{E}_{n}$ exceeds $k_{\mathrm{B}} T$ for some $n$ ) on $\epsilon$ and $\mathcal{E}_{0}(0)$. The black line is a least-squares fit to the data. The numerical simulation becomes more sensitive the more energy is put into the system, which explains the increased spread of the data points as $\mathcal{E}_{0}(0)$ increases.

To include dispersion, and to reach a proper thermal equilibrium state, fluctuation corrections must be included. As shown in Appendix B, this leads to the following FokkerPlanck equation for the reduced probability density,

$$
\partial_{\tau} p_{0}=\alpha \partial_{\mathcal{E}} \mathcal{E}^{2}\left[p_{0}+\left(\epsilon \mathcal{D} \gamma / \omega_{0}\right) \partial_{\mathcal{E}} p_{0}\right] \text {. }
$$

Noting that $\left(\epsilon \mathcal{D} \gamma / \omega_{0}\right)=2 k_{\mathrm{B}} T /\left(M \omega_{0}^{2} L^{2}\right)$, we see that this FPE also gives the proper thermal equilibrium stationary solution $p_{0}(\tau \rightarrow \infty) \propto \exp \left(-M \omega_{0}^{2} q_{0}^{2} / 2 k_{\mathrm{B}} T\right)$.

\section{MULTIMODE DYNAMICS, THERMALIZATION}

While the qualitative behavior of the dynamics remain unchanged by incorporating more flexural modes, a quantitative change takes place. If the higher-lying modes are initially at rest, we find that exciting the system in only the fundamental mode rapidly causes the higher modes to be thermalized. Once in thermal equilibrium, they provide additional channels for energy dissipation from the fundamental mode.

The thermalization of the higher modes stem from the stochastic additive noise term in Eq. (3). As initially $\mathcal{E}_{0} \gg$ $\mathcal{E}_{n>0}$, the transient behavior is described by $\ddot{q}_{n}+\omega_{n}^{2} q_{n} \approx$ $\epsilon \omega_{0}^{2} q_{0} \phi_{n} \phi_{0} \propto \epsilon \sqrt{\mathcal{E}_{0}}, n>0$. To a first approximation, we would thus expect the energy of the higher-lying modes to have a transient behavior $\left\langle\mathcal{E}_{n}\right\rangle \sim \epsilon^{2} \mathcal{E}_{0} t$. In order to investigate this, we define the thermalization time $\tau_{\text {therm }}$ as the time when the energy of an excited mode first exceeds the thermal energy. Simulations were made at a constant temperature but for values of $\epsilon$ and $\mathcal{E}_{0}(0)$ ranging over several orders of magnitude; the resulting values for $\tau_{\text {therm }}$ are shown in Fig. 5. The data have been fitted to a model $\tau_{\text {therm }} \propto\left(\epsilon^{a} \mathcal{E}_{0}\right)^{b}$, and the exponent $a=1.86(b \approx 1.2)$ was determined by minimizing the sum of squared residuals of the linear fit shown as a black line in Fig. 5. The slight deviation from the theoretical value of

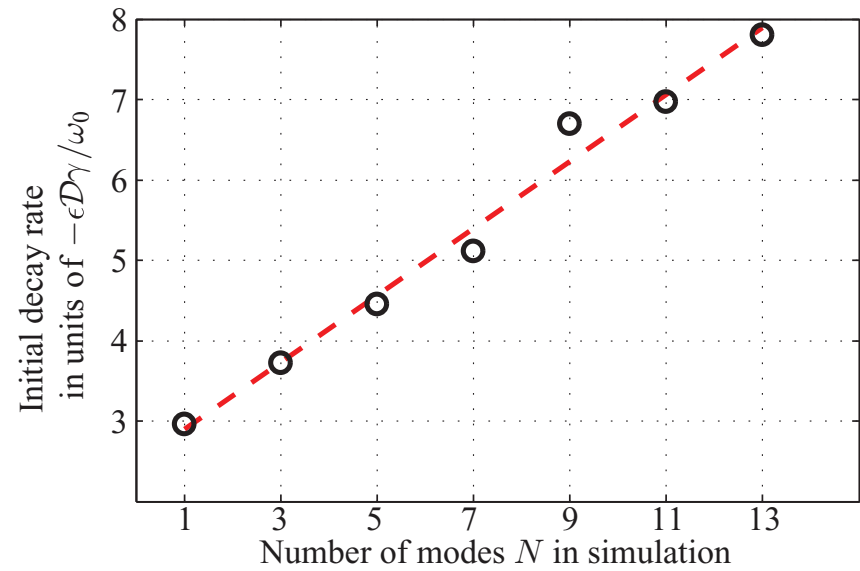

FIG. 6. (Color online) Slope of the decay of the fundamental mode during the initial, linear regime, as a function of the number of modes $N$ included in the simulation; black circles are data points while the red dashed line is a linear fit. The values have been divided by $-\epsilon \mathcal{D} \gamma / \omega_{0}$ to show the agreement with the theoretical case (10). As more excited modes are included, more decay channels are opened and the rate of decay increases.

$a=2$ is likely due to the present definition of $\tau_{\text {therm }}$, which will always be smaller than the time taken for all higher-lying modes to reach $k_{\mathrm{B}} T$.

The parameters $\epsilon$ and $\mathcal{E}_{0}(0)$ are straightforward to vary in experiments, e.g., by using different-sized nanoclusters as adsorbates and by varying the driving force used before beginning the ring-down. Hence, the linear dependence found in Fig. 5 should be possible to verify experimentally.

In addition, we note that for $\mathcal{E}_{0}(0) \gg k_{\mathrm{B}} T$, the thermalization of excited modes occur on a time scale much shorter than the decay of the fundamental mode. Consequently, on the time scale relevant for studying the ring-down of the resonator, it is a good approximation to assume that all excited modes are in thermal equilibrium.

The fact that the mode coupling strength and energy transfer between modes are determined only by the energy in the modes is further corroborated by considering the decay rate of the fundamental mode energy once the higher modes have thermalized. As shown in Fig. 6, for each additional mode we include in the simulation, an additional channel for energy transfer away from the fundamental mode is made available and the decay rate (initially) increases linearly with the number of added modes. As each individual mode has the same energy $\sim k_{\mathrm{B}} T$, each mode contributes an equal amount to the fundamental mode dissipation.

Clearly, there must be a cutoff at which this is no longer true. Such a cutoff can be estimated by noting that due to the influence of the fundamental mode, the dissipation of energy from the fundamental mode is associated with adsorbate dynamics occurring on a time scale $\omega_{0}^{-1}$ corresponding to a diffusion length scale of $\sqrt{2 \pi D / \omega_{0}}$. If this length is larger than half the wavelength of the $n$th mode, the effective coupling to this mode will average to zero and not contribute to dissipation. The wavelength is $\lambda_{n}=2 L /(n+1)$, giving the cutoff condition that only modes with $n \lesssim n_{\max } \approx \sqrt{2 / \pi \mathcal{D}}$ will contribute to the dissipation of the fundamental mode. For the parameter values used in Fig. 6 we find $n_{\max } \approx 20$. 


\section{CONCLUSIONS}

We have studied the effect of a diffusing adsorbed particle on a vibrating one-dimensional nanomechanical resonator, initially excited in its fundamental flexural mode. Studying the free ring-down of the mode, focusing on the energy transfer induced by the diffusion we find that there are two effects that cause vibrational energy to dissipate.

First, the inertial force exerted on the adsorbed particle causes short-time correlations between adsorbate motion and flexural vibrations. For large initial amplitudes such that $m \omega_{0}^{2} q_{0}^{2} / k_{\mathrm{B}} T \gg 1$, the particle is trapped at the antinode of vibration, and the decay of vibrational energy is linear in time, approximately given by expression (10). For lower amplitudes, when the particle diffuses freely along the resonator, the decay takes the same form as that of a nonlinearly damped oscillator according to Eq. (15).

Second, the diffusing particle also provides a stochastic coupling between different flexural vibration modes. This stochastic coupling provides an additional channel for energy transfer from the fundamental mode for each added flexural mode. This second mechanism can further significantly lower the ring-down time of the fundamental mode and causes rapid thermalization among the higher modes. The dissipation rate due to the mode coupling is linear in the number of included excited modes (up to a parameter-dependent cutoff), meaning that a single-mode treatment of a resonator is qualitatively but not quantitatively correct. The exact thermalization rate of the excited modes depends on adsorbate mass $(\epsilon)$, device geometry $\left(\omega_{0}\right)$, and initial amplitude $\left[\mathcal{E}_{0}(0)\right]$; parameters that are readily accessible in experiments.

With recent advances in readout of the real-time evolution of nanomechanical oscillators $[19,20]$ together with the ability to deposit individual particles on ultrahigh- $Q$ carbon nanotube resonators [5], the proposed effects should be possible to observe experimentally. These result also have bearing on the numerical analysis in Ref. [21] which showed a dramatic change in $Q$ factor for an Au cluster deposited on a graphene resonator at the onset of particle diffusion. Finally, the existence of a trapping regime where adsorbate diffusion is suppressed implies that mass sensing experiments above cryogenic temperatures may be possible, given that the resonator is driven strongly enough.

\section{ACKNOWLEDGMENTS}

We acknowledge financial support from the Swedish Research Council VR (A.I.), the The Foundation for Strategic Research SSF (C.E.) as well as the European Union through Grant No. 246026 (A.I., C.E.) and the GRAPHENE Flagship (C.E.).

\section{APPENDIX A: DETERMINING THE $\omega_{n}$ and $\phi_{n}(X)$}

Here, we derive the flexural eigenmodes and eigenfrequencies for the unperturbed resonator. The equation of motion corresponding to the Lagrangian (1) is

$$
\rho \ddot{w}-\sigma \partial_{X}^{2} w+\kappa \partial_{X}^{4} w=0 .
$$

Defining $\phi_{n}(X)$ and $\omega_{n}$ through $w(X, t)=e^{-i \omega_{n} t} \phi_{n}(X)$, we find that the eigenmodes satisfy the equation

$$
-\rho \omega_{n}^{2} \phi_{n}-\sigma \phi_{n}^{\prime \prime}+\kappa \phi_{n}^{\prime \prime \prime \prime}=0 .
$$

The corresponding characteristic equation is

$$
\kappa k^{4}-\sigma k^{2}-\rho \omega_{n}^{2}=0
$$

with roots $\pm k_{n}^{+}, \pm i k_{n}^{-}$, where

$$
k_{n}^{ \pm}=\sqrt{\sqrt{\frac{\sigma^{2}}{4 \kappa^{2}}+\frac{\rho \omega_{n}^{2}}{\kappa}} \pm \frac{\sigma}{2 \kappa}}, \quad n=0,1,2, \ldots
$$

Hence, the eigenmodes can be written as

$$
\begin{aligned}
\phi_{2 n}= & A_{2 n} \operatorname{ch} k_{2 n}^{+}\left(X-\frac{L}{2}\right)+A_{2 n}^{\prime} \cos k_{2 n}^{-}\left(X-\frac{L}{2}\right), \\
\phi_{2 n+1}= & B_{2 n+1} \operatorname{sh} k_{2 n+1}^{+}\left(X-\frac{L}{2}\right) \\
& +B_{2 n+1}^{\prime} \sin k_{2 n+1}^{-}\left(X-\frac{L}{2}\right),
\end{aligned}
$$

where the boundary conditions $\phi_{n}(0)=\phi_{n}(L)=\phi_{n}^{\prime}(0)=$ $\phi_{n}^{\prime}(L)=0$ have been used to divide the $\phi_{n}$ into even and odd sets of eigenmodes. Similarily, we find that the eigenfrequencies $\omega_{n}$ are determined from the equation

$$
\frac{k_{n}^{\mp}}{k_{n}^{ \pm}}= \pm \frac{\tan k_{n}^{-} \frac{L}{2}}{\tanh k_{n}^{+} \frac{L}{2}},
$$

where upper/lower signs correspond to odd/even $n$. A good approximation for the ratio $\omega_{n} / \omega_{0}$ is $(2 n+1)^{2} / 9$. Finally, the integration constants $A_{n}$ and $B_{n}$ are determined. The boundary conditions demand that

$$
A_{n}^{\prime}=-\frac{\cosh k_{n}^{+} \frac{L}{2}}{\cos k_{n}^{-} \frac{L}{2}} A_{n}, \quad B_{n}^{\prime}=-\frac{\sinh k_{n}^{+} \frac{L}{2}}{\sin k_{n}^{-} \frac{L}{2}} B_{n}
$$

whereas the normalization condition $\int \mathrm{d} X \phi_{m}^{\dagger} \phi_{n}=L \delta_{m n}$ determines

$$
\begin{aligned}
& \left|A_{n}\right|^{2}=2\left[1+\frac{\operatorname{sh} k_{n}^{+} L}{k_{n}^{+} L}+\frac{\operatorname{ch}^{2} k_{n}^{+} \frac{L}{2}}{\cos ^{2} k_{n}^{-} \frac{L}{2}}\left(1+\frac{\sin k_{n}^{-} L}{k_{n}^{-} L}\right)\right]^{-1}, \\
& \left|B_{n}\right|^{2}=2\left[1-\frac{\operatorname{sh} k_{n}^{+} L}{k_{n}^{+} L}-\frac{\operatorname{sh}^{2} k_{n}^{+} \frac{L}{2}}{\sin ^{2} k_{n}^{-} \frac{L}{2}}\left(1-\frac{\sin k_{n}^{-} L}{k_{n}^{-} L}\right)\right]^{-1} .
\end{aligned}
$$

The final undetermined phase is chosen so that the eigenfunctions $\phi_{n}$ are real.

\section{APPENDIX B: FORMAL PERTURBATION THEORY}

The perturbation theory sketched in Sec. III B can be put on more formal grounds. In this appendix we derive the reduced FPE by means of the methods in Ref. [24]. Introducing the variable $v=\omega_{0} t+\theta$, the FPE reads

$$
\begin{aligned}
\partial_{\tau} p= & \epsilon \phi_{0}^{2} \mathcal{E} \sin (2 \nu) \partial_{\mathcal{E}} p\left(\frac{\epsilon \phi_{0}^{2}}{2}[1+\cos (2 \nu)]-1\right) \partial_{\nu} p \\
& -\frac{\omega_{0} \mathcal{E}}{2 \gamma}[1+\cos (2 \nu)] \partial_{\xi}\left[p \partial_{\xi} \phi^{2}\right]+\mathcal{D} \partial_{\xi}^{2} p .
\end{aligned}
$$

Upon expanding $p=\sum_{n} p_{n}(\mathcal{E}, \xi, \tau) e^{2 i n v}$ and introducing the vector $\mathbf{p}=\left[\ldots, p_{1}, p_{0}, p_{1}, \ldots\right]^{\perp}$, the FPE can be 
rewritten as

$$
\partial_{\tau} \mathbf{p}=\epsilon \hat{L}_{1} \mathbf{p}+\frac{\mathcal{D}}{2} \hat{L}_{2} \mathbf{p}
$$

where $\hat{L}_{1} \mathbf{p}=\phi_{0}^{2}\left[\mathcal{E} \hat{A} \partial_{\mathcal{E}}+i \hat{B} \hat{N}\right] \mathbf{p}$ and

$$
\hat{L}_{2} \mathbf{p}=-4 i \mathcal{D}^{-1} \hat{N} \mathbf{p}-\frac{\omega_{0} \mathcal{E}}{2 \mathcal{D} \gamma} \hat{B} \partial_{\xi}\left[\mathbf{p} \partial_{\xi} \phi^{2}\right]+\partial_{\xi}^{2} \mathbf{p} .
$$

The matrices $\hat{A}, \hat{B}, \hat{N}$, have components $A_{m, n}=$ $(2 i)^{-1}\left[\delta_{m, n+1}-\delta_{m, n-1}\right], B_{m, n}=\delta_{m, n}+2^{-1}\left[\delta_{m, n+1}+\delta_{m, n-1}\right]$, and $N_{m, n}=n \delta_{m, n}$.

Expanding in eigenmodes of the operator $\hat{L}_{2}$, as $\mathbf{p}=$ $\sum_{n} \beta_{n}(\mathcal{E}, \tau) \mathbf{v}_{n}(\xi, \mathcal{E})$, where $\hat{L}_{2} \mathbf{v}_{n}=-\mu_{n}(\mathcal{E}) \mathbf{v}_{n}$ yields

$$
\left(\partial_{\tau}+\frac{\mathcal{D} \mu_{n}}{2}\right) \beta_{n}=\epsilon \sum_{m}\left\langle\mathbf{w}_{n}, \hat{L}_{1} \beta_{m} \mathbf{v}_{m}\right\rangle
$$

The inner product is here defined as $\langle\mathbf{u}, \mathbf{v}\rangle \equiv \int d \xi \mathbf{u}^{\dagger} \mathbf{v}$ and the right eigenvectors $\mathbf{w}_{n}$ satisfy the adjoint equation $L_{2}^{*} \mathbf{w}_{n}=$ $-\mu_{n}^{*} \mathbf{w}_{n}$ with

$$
L_{2}^{*}=4 i \mathcal{D}^{-1} \hat{N}+\frac{\omega_{0} \mathcal{E}}{2 \mathcal{D} \gamma} \hat{B}\left(\partial_{\xi} \phi_{0}^{2}\right) \partial_{\xi}+\partial_{\xi}^{2} .
$$

For time scales $\tau>\mathcal{D}^{-1}$, we can make the approximation

$$
\begin{aligned}
& \partial_{\tau} \beta_{0}=\epsilon \sum_{n}\left\langle\mathbf{w}_{0}, \hat{L}_{1} \beta_{n} \mathbf{v}_{n}\right\rangle, \\
& \beta_{n \geqslant 1} \approx \frac{2 \epsilon}{\mathcal{D} \mu_{n}}\left\langle\mathbf{w}_{n}, \hat{L}_{1} \beta_{0} \mathbf{v}_{0}\right\rangle .
\end{aligned}
$$

Combining the two gives

$$
\begin{aligned}
\partial_{\tau} \beta_{0}= & \epsilon\left\langle\mathbf{w}_{0}, \hat{L}_{1} \beta_{0} \mathbf{v}_{0}\right\rangle \\
& +\frac{2 \epsilon^{2}}{\mathcal{D}} \sum_{n \geqslant 1}\left\langle\mathbf{w}_{0}, \hat{L}_{1}\left(\mu_{n}^{-1}\left\langle\mathbf{w}_{n}, \hat{L}_{1} \beta_{0} \mathbf{v}_{0}\right\rangle\right) \mathbf{v}_{n}\right\rangle .
\end{aligned}
$$

\section{Perturbation theory for Eigenvectors}

The eigenvectors of $\hat{L}_{2}$ cannot obtained exactly. However, if the parameter $\eta \equiv \frac{\omega_{0} \mathcal{E}}{2 \mathcal{D} \gamma} \ll 1$ we can find them perturbatively to first order in $\eta$. Each eigenvector-eigenvalue pair has composite indices $(n, m)$ and is to first order given by

$$
\begin{aligned}
& \mathbf{v}_{n m} \approx \mathbf{v}_{n m}^{(0)}+\frac{\omega_{0} \mathcal{E}}{2 \mathcal{D} \gamma} \sum_{p q \neq m n} \frac{\left\langle\mathbf{w}_{p q}^{(0)}, \hat{B} \partial_{\xi} \mathbf{v}_{n m}^{(0)} \partial_{\xi} \phi_{0}^{2}\right\rangle}{\mu_{n m}^{(0)}-\mu_{p q}^{(0)}} \mathbf{v}_{p q}^{(0)}, \\
& \mathbf{w}_{n m} \approx \mathbf{w}_{n m}^{(0)}-\frac{\omega_{0} \mathcal{E}}{2 \mathcal{D} \gamma} \sum_{p q \neq m n} \frac{\left\langle\mathbf{w}_{p q}^{(0)}, \hat{B}\left(\partial_{\xi} \phi_{0}^{2}\right) \partial_{\xi} \mathbf{v}_{n m}^{(0)}\right\rangle}{\left(\mu_{n m}^{(0)}-\mu_{p q}^{(0)}\right)^{*}} \mathbf{w}_{p q}^{(0)}, \\
& \mu_{n m} \approx \mu_{n m}^{(0)}+\frac{\omega_{0} \mathcal{E}}{2 \mathcal{D} \gamma}\left\langle\mathbf{w}_{n m}^{(0)}, \hat{B} \partial_{\xi} \mathbf{v}_{n m}^{(0)} \partial_{\xi} \phi_{0}^{2}\right\rangle .
\end{aligned}
$$

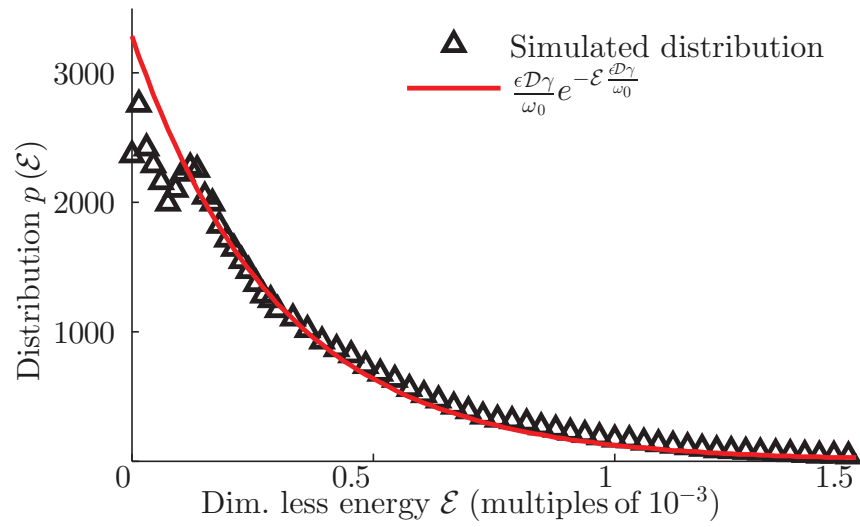

FIG. 7. (Color online) Equilibrium distribution $p_{\text {st. }}(\mathcal{E})$. (Black triangles) Distribution obtained from numerical simulation of Equations. (7)-(9) after relaxation. (Red line) The distribution $p(\mathcal{E})=$ $\left(\epsilon \mathcal{D} \gamma / \omega_{0}\right) \exp \left(-\mathcal{E}\left(\epsilon \mathcal{D} \gamma / \omega_{0}\right)\right)$.

The unperturbed eigenvalues are $\mu_{n m}^{(0)}=n^{2} \pi^{2}+4 i \mathcal{D}^{-1} m$ and the corresponding eigenvector has the $k$ th component

$$
\left[\mathbf{v}_{n m}^{(0)}\right]_{k}=\left[\mathbf{w}_{n m}^{(0)}\right]_{k}=\left[\sqrt{2} \cos (n \pi \xi)+\delta_{n, 0}(1-\sqrt{2})\right] \delta_{k, m} .
$$

\section{Derivation of FPE for reduced density $\beta_{0}(\mathcal{E}, \tau)$}

To obtain the FPE for the slowly varying coefficient $\beta_{0}(\mathcal{E}, \tau)$, corresponding to the probability density $p_{0}(\mathcal{E}, \tau)$ in the main text, we first observe that

$$
\begin{aligned}
& \left\langle\mathbf{w}_{0}, \mathcal{L}_{1} F(\mathcal{E}) \mathbf{v}_{n m}\right\rangle \\
& \quad=(i / 2) \partial_{\mathcal{E}}\left[\mathcal{E} F(\mathcal{E}) \int d \xi \phi_{0}^{2}\left(\left[\mathbf{v}_{n m}\right]_{1}-\left[\mathbf{v}_{n m}\right]_{-1}\right)\right]
\end{aligned}
$$

for an arbitrary function $F(\mathcal{E})$. With $F(\mathcal{E})=\beta_{0}(\mathcal{E})$ we then recover the expression in Eq. (13),

$$
\epsilon\left\langle\mathbf{w}_{0}, \hat{L}_{1} \beta_{0} \mathbf{v}_{0}\right\rangle=\epsilon \frac{2 \omega_{0}}{\mathcal{D} \gamma} \sum_{n} f_{n}^{2} \frac{\lambda_{n}}{\left(\lambda_{n}\right)^{2}+16} \partial_{\mathcal{E}}\left[\mathcal{E}^{2} \beta_{0}\right] .
$$

For the fluctuation correction, the lowest-order term arises from considering only the unperturbed eigenvectors,

$$
\begin{aligned}
& 2 \epsilon^{2}\left\langle\mathbf{w}_{0}^{0}, \hat{L}_{1}\left(\left(\mu_{n m}^{0}\right)^{-1}\left\langle\mathbf{w}_{n m}^{0}, \hat{L}_{1} \beta_{0} \mathbf{v}_{0}^{0}\right\rangle\right) \mathbf{v}_{n m}^{0}\right\rangle \\
& =2 \epsilon^{2} \sum_{n} \frac{f_{n}^{2} \lambda_{n}}{\lambda_{n}^{2}+16} \partial_{\mathcal{E}} \mathcal{E}^{2} \partial_{\mathcal{E}} \beta_{0} \\
& =\alpha\left(\frac{\epsilon \mathcal{D} \gamma}{\omega_{0}}\right) \partial_{\mathcal{E}} \mathcal{E}^{2} \partial_{\mathcal{E}} \beta_{0} .
\end{aligned}
$$

Hence, to lowest order in $\mathcal{E}$ and to second order in $\epsilon$, the FPE reads

$$
\partial_{\tau} \beta_{0}=\alpha \partial_{\mathcal{E}} \mathcal{E}^{2}\left(\beta_{0}+\left(\frac{\epsilon \mathcal{D} \gamma}{\omega_{0}}\right) \partial_{\mathcal{E}} \beta_{0}\right) .
$$

The stationary solution to this equation is the equilibrium distribution $p(\mathcal{E})=\left(\epsilon \mathcal{D} \gamma / \omega_{0}\right) \exp \left[-\mathcal{E}\left(\epsilon \mathcal{D} \gamma / \omega_{0}\right)\right]$ which we also confirm by direct numerical simulation (see Fig. 7). 
[1] J. L. Arlett, E. B. Myers, and M. L. Roukes, Nat. Nanotechnol. 6, 203 (2011).

[2] M. S. Hanay, S. Kelber, A. K. Naik, D. Chi, S. Hentz, E. C. Bullard, E. Colinet, L. Duraffourg, and M. L. Roukes, Nat. Nanotechnol. 7, 602 (2012).

[3] B. Lassagne, D. Garcia-Sanchez, A. Aguasca, and A. Bachtold, Nano Lett. 8, 3735 (2008).

[4] K. Jensen, K. Kim, and A. Zettl, Nat. Nanotechnol. 3, 533 (2008).

[5] J. Chaste, A. Eichler, J. Moser, G. Ceballos, R. Rurali, and A. Bachtold, Nat. Nanotechnol. 7, 301 (2012).

[6] J. Atalaya, A. Isacsson, and M. I. Dykman, Phys. Rev. B 83, 045419 (2011).

[7] M. I. Dykman, M. Khasin, J. Portman, and S. W. Shaw, Phys. Rev. Lett. 105, 230601 (2010).

[8] J. Atalaya, A. Isacsson, and M. I. Dykman, Phys. Rev. Lett. 106, 227202 (2011).

[9] J. Atalaya, J. Phys.: Condens. Matter 24, 475301 (2012).

[10] Y. T. Yang, C. Callegari, X. L. Feng, and M. L. Roukes, Nano Lett. 11, 1753 (2011).

[11] R. Lifshitz and M. L. Roukes, Phys. Rev. B 61, 5600 (2000).

[12] M. C. Cross and R. Lifshitz, Phys. Rev. B 64, 085324 (2001).

[13] I. Wilson-Rae, Phys. Rev. B 77, 245418 (2008).
[14] K. Eom, H. S. Park, D. S. Yoon, and T. Kwon, Phys. Rep. 503, 115 (2011).

[15] A. Eichler, J. Moser, J. Chaste, M. Zdrojek, I. Wilson-Rae, and A. Bachtold, Nat. Nanotech. 6, 339 (2011).

[16] A. Croy, D. Midtvedt, A. Isacsson, and J. M. Kinaret, Phys. Rev. B 86, 235435 (2012).

[17] D. Midtvedt, A. Croy, A. Isacsson, Z. Qi, and H. S. Park, Phys. Rev. Lett. 112, 145503 (2014).

[18] A. W. Barnard, V. Sazonova, A. M. van der Zande, and P. L. McEuen, Proc. Natl. Acad. Sci. USA 109, 19093 (2012).

[19] H. B. Meerwaldt, S. R. Johnston, H. S. J. van der Zant, and G. A. Steele, Appl. Phys. Lett. 103, 053121 (2013).

[20] R. van Leeuwen, A. Castellanos-Gomez, G. A. Steele, H. S. J. van der Zant, and W. J. Venstra, Appl. Phys. Lett. 105, 041911 (2014).

[21] J.-W. Jiang, B.-S. Wang, H. S. Park, and T. Rabczuk, Nanotechnology 2, 02501 (2014).

[22] L. D. Landau and E. M. Lifshitz, Theory of Elasticity, 3rd ed. (Elsevier Butterworth Heinmann, New York, 1986).

[23] R. Mannella and V. Palleschi, Phys. Rev. A 40, 3381 (1989).

[24] H. Risken, The Fokker-Planck Equation: Methods of Solution and Applications, Springer Series in Synergetics Vol. 18, 2nd ed. (Springer-Verlag, New York, 1996). 